\title{
Acoustoelastic pure-displacement elements based on Biot's poromechanics theory
}

\author{
V.L. Guadalupe ${ }^{\mathrm{a}, *}$, C. Militello ${ }^{\mathrm{a}}$, M. Recuero ${ }^{\mathrm{b}}$ \\ a Departamento de Física Fundamental, Experimental, Electrónica y Sistemas, Universidad de La Laguna, Calle Astrofisico Sánchez S/N, 28205 La Laguna, Tenerife, Spain \\ ${ }^{\mathrm{b}}$ Grupo de Investigación en Instrumentación y Acústica Aplicada (I2A2), Universidad Politécnica de Madrid (UPM), Ctra. De Valencia km 7 - Campus Sur, 28031 Madrid, Spain
}

A R T I C L E I N F O

Keywords:

Porous elements

Displacement formulation

Spurious modes

Porous beam

Porous tetrahedron

\begin{abstract}
A B S T R A C T
From the elastic energy contained in a displacement based porous material the general form of the stiffness and mass matrices are obtained. If both fields can be expanded with equal order polynomials the general form is further simplified and it is shown that the coupling stiffness reduces to the one arising to compute volume changes in an elastic medium. A plane beam and a four node tetrahedron are developed. To avoid spurious rotational modes appearance in the tetrahedron fluid a penalty formulation is used. The effect of the penalty factor in matrix conditioning is analysed. Elements dynamic behaviour is compared.
\end{abstract}

\section{Introduction}

A framework for constructing porous material element matrices based on Biot's theory [1] is presented. The development relies on the paper Elastic Waves in Porous Solids I, by Biot and Willis [2]. It is well-known that Biot renamed the coefficients over the years, but the nomenclature used for the elastic constants and stress definitions of this work persisted. From the elastic energy accumulated in an isotropic porous material that undergoes a stress state the general form of the stiffness matrix is obtained assuming displacements fields for both the porous matrix and the filling fluid. The displacements fields are noted by vectors $\mathbf{u}$ and $\mathbf{U}$ respectively. If both displacements fields are developed with the same polynomial order the stiffness matrix is further simplified.

Once the framework is obtained and discussed, two elements are presented: a porous $2 \mathrm{D}$ jacketed beam and a four-node tetrahedron. The planar beam does not present spurious rotational modes problems.

A problem with the $(\mathbf{u}, \mathbf{U})$ formulations is that $\mathbf{U}$ in the finite element expansion is a rotational vector field, but the energy computed for an acoustic fluid does not take into account rotational effects. Because of this, the model computes low-energy rotational modes, called spurious modes. They are spurious for an irrotational fluid formulation, but they are correct for the displacement field used. Many authors have addressed this by introducing a fictitious rotational energy for the fluid with a penalty factor $[3,4]$. The serious drawback is that the penalty factor depends on user knowledge or experience, ranging from 100 to 10,000 .

\footnotetext{
* Corresponding author. Tel.: +34 922 318303; fax: +34 922318228.

E-mail address: vlsuarez@ull.es (V.L. Guadalupe).
}

There are other two solutions. One involves using a RaviartThomas displacement expansion for the fluid [5]. Raviart-Thomas expansions produce irrotational fields, but they have the problem that degrees of freedom are placed at the midsides or at the centre-face of finite elements, making it difficult to interface them with standard elements [6]. The other solution was introduced in [7], where, by the use of the Parameterized Variational Principles [8] and the Individual Element Test [9], a weakly irrotational constraint is introduced in the original formulation. It also required the introduction of an additional parameter, but an energy balance allowed the authors to compute it in closed form as a function of element size. The formula can be included in the code and requires no user interaction.

A Patch Test, combined with the experiments proposed by Biot and Willis to compute porous material properties, provides a framework for discussing minimum boundary conditions. A shear test is used to assess the effect of the parameter in the coupled stiffness matrix condition.

A literature survey for other porous material formulations and its effect on boundary conditions can be found in [10-15].

The present work is organized in the following sections. In Section 2 the general scheme to construct the stiffness and mass matrices of the porous material is developed. Section 3 deals with the construction of a $2 \mathrm{D}$ porous beam element based on the general scheme. Then, in Section 4, a four node linear tetrahedron is constructed. Because it needs to be stabilized introducing a penalty factor the effect of the factor in numerical conditioning and element consistency are analysed. Finally, in Section 5, a simple bending test is performed to check convergence of both models to the same bending frequencies and to show solid and fluid fields behaviour. 


\section{The porous element matrices}

\subsection{The stiffness matrix}

In Biot's theory, a porous material is made of an elastic matrix of porous material and an irrotational acoustic fluid filling it. The forces acting over the surface of a reference volume will simultaneously generate a stress field $(\sigma, \tau)$ over the solid and a stress field $s$ over the fluid. The elastic constants are $N, A, Q$ and $R$. In matrix form the stress-strain relationships can be written as

$$
\left\{\begin{array}{c}
\sigma_{x} \\
\sigma_{y} \\
\sigma_{z} \\
\tau_{x y} \\
\tau_{y z} \\
\tau_{z x} \\
s
\end{array}\right\}=\left[\begin{array}{ccccccc}
2 N+A & A & A & 0 & 0 & 0 & Q \\
A & 2 N+A & A & 0 & 0 & 0 & Q \\
A & A & 2 N+A & 0 & 0 & 0 & Q \\
0 & 0 & 0 & N & 0 & 0 & 0 \\
0 & 0 & 0 & 0 & N & 0 & 0 \\
0 & 0 & 0 & 0 & 0 & N & 0 \\
Q & Q & Q & 0 & 0 & 0 & R
\end{array}\right\}\left\{\begin{array}{c}
e_{x} \\
e_{y} \\
e_{z} \\
\gamma_{x y} \\
\gamma_{y z} \\
\gamma_{z x} \\
\varepsilon
\end{array}\right\}
$$

where

$$
\boldsymbol{\sigma}=\mathbf{D e}=\left[\begin{array}{cc}
D_{s s} & D_{s f} \\
D_{s f}^{T} & D_{f f}
\end{array}\right]\left[\begin{array}{l}
\mathbf{e}_{s} \\
\mathbf{e}_{f}
\end{array}\right]
$$

Strains $e_{x}, e_{y}, e_{z}, \gamma_{x y}, \gamma_{y z}$ and $\gamma_{z x}$ and can be computed from the displacement field assumed for the solid matrix. Strain measure $e$ is its unitary volume change. For the fluid, the only strain measure is its unitary volume change $\varepsilon$

$$
\begin{aligned}
& e=e_{x}+e_{y}+e_{z} \\
& \varepsilon=\varepsilon_{x}+\varepsilon_{y}+\varepsilon_{z}
\end{aligned}
$$

Strains fields are computed from the displacements fields. The porous matrix displacement vector $\mathbf{u}$ has components $\left(u_{x}, u_{y}, u_{z}\right)$ and the fluid displacement vector $\mathbf{U}$ has components $\left(U_{x}, U_{y}, U_{z}\right)$.

In keeping with Biot, the potential elastic energy inside a porous element can be obtained from:

$$
\Pi=\frac{1}{2} \int_{V} \boldsymbol{\sigma}^{\mathbf{T}} \mathbf{e} d V=\frac{1}{2} \int_{V} \mathbf{e}^{\mathbf{T}} \mathbf{D e} d V=\frac{1}{2} \int_{V}\left[\begin{array}{l}
\mathbf{e}_{s} \\
\mathbf{e}_{f}
\end{array}\right]^{T}\left[\begin{array}{cc}
D_{s s} & D_{s f} \\
D_{s f}^{T} & D_{f f}
\end{array}\right]\left[\begin{array}{l}
\mathbf{e}_{s} \\
\mathbf{e}_{f}
\end{array}\right] d V
$$

Following standard finite element technology procedures, a general shape for the porous material stiffness matrix is:

$$
\mathbf{K}_{\text {porous }}=\left[\begin{array}{ll}
\mathbf{K}_{s s} & \mathbf{K}_{s f} \\
\mathbf{K}_{s f}^{T} & \mathbf{K}_{f f}
\end{array}\right]
$$

where

$$
\begin{aligned}
\mathbf{K}_{s s} & =\int_{V} \mathbf{B}_{s}^{T} \mathbf{D}_{s s} \mathbf{B}_{s} d V \\
\mathbf{K}_{s f} & =\int_{V} \mathbf{B}_{s}^{T} \mathbf{D}_{s f} \mathbf{B}_{f} d V=\mathbf{K}_{f s}^{T} \\
\mathbf{K}_{f f} & =\int_{V} \mathbf{B}_{s}^{T} \mathbf{D}_{f f} \mathbf{B}_{f} d V
\end{aligned}
$$

The $\mathbf{B} s$ are the strain-nodal displacement operators. The energy for the coupling inside one element is obtained from

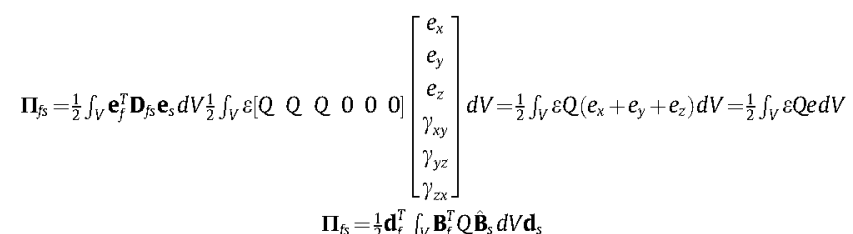

If the same polynomial expansion is chosen for both fields, then $\hat{\mathbf{B}}_{s}=\mathbf{B}_{f}$ and the coupling matrix becomes a symmetrical matrix. Moreover, extracting the elastic constants reveals that $\mathbf{K}_{s f}=\mathbf{K}_{f f}$. Finally, only two different stiffness matrices are needed to compute the stiffness matrix of the isotropic porous media, one for the solid porous matrix and one for the unit change in fluid volume:

$\mathbf{K}_{\text {porous }}=\left[\begin{array}{cc}\mathbf{K}_{s s} & Q \mathbf{K}_{f f} \\ Q \mathbf{K}_{f f} & R \mathbf{K}_{f f}\end{array}\right]$

\subsection{The mass matrix}

Biot proposed the following form for the kinetic energy of a porous material:

$2 T_{B}=\rho_{11} \dot{\mathbf{u}}^{T} \cdot \dot{\mathbf{u}}+2 \rho_{12} \dot{\mathbf{u}}^{T} \cdot \dot{\mathbf{U}}+\rho_{22} \dot{\mathbf{U}}^{T} \cdot \dot{\mathbf{U}}$

This is valid for a statistically isotropic material with no preferred directions. The solid displacement, velocity and accelerations are indicated by $\mathbf{u}, \dot{\mathbf{u}}$, $\ddot{\mathbf{u}}$ respectively. The fluid counterparts are $\mathbf{U}, \dot{\mathbf{U}}, \ddot{\mathbf{U}}$. To explain the meaning of the $\rho$ coefficients, we compute the Lagrangian in the $x$ direction:

$$
\begin{aligned}
& \frac{\partial}{\partial t}\left(\frac{\partial T_{B}}{\partial \dot{u}_{x}}\right)=\rho_{11} \ddot{u}_{x}+\rho_{12} \ddot{U}_{x} \\
& \frac{\partial}{\partial t}\left(\frac{\partial T_{B}}{\partial \dot{U}_{x}}\right)=\rho_{12} \ddot{u}_{x}+\rho_{22} \ddot{U}_{x}
\end{aligned}
$$

Assuming that $u_{x}=U_{x}$ in Eq. (9)

$$
2 T_{B}=\left(\rho_{11}+2 \rho_{12}+\rho_{22}\right) \dot{u}_{x}^{2}
$$

then,

$\left(\rho_{11}+2 \rho_{12}+\rho_{22}\right)=\rho$

In Eq. (12), $\rho$ is the porous material's complete density. It can be computed from the properties of the constituents and the porosity. The density contribution of each component to a unit volume for the solid matrix of a porous material is:

$\rho_{1}=(1-\beta) \rho_{s}$

and for the fluid it is

$\rho_{2}=\beta \rho_{f}$

where $\beta$ is the porosity and $\rho_{s}$ and $\rho_{f}$ the density of the raw materials. Finally, Biot concluded that:

$\rho_{1}=\rho_{11}+\rho_{12}$

$\rho_{2}=\rho_{12}+\rho_{22}$

If $\rho_{12}$ depends on frequency, then $\rho_{11}$ must as well, keeping $\rho_{1}$ free from frequency dependence. Biot introduced the concept of an apparent mass so that $\rho_{12}=-\rho_{a}$. It must be noted that because $\rho_{a}$ is defined as a positive quantity, then $\rho_{12} \leqslant 0$. Finally:

$\rho_{11}=\rho_{1}+\rho_{a}$

$\rho_{22}=\rho_{2}+\rho_{a}$

The apparent mass is assumed to change with frequency. To make sure that the defined kinetic energy is positive, the following relationships among coefficients must hold:

$\rho_{11} \geqslant 0 \quad \rho_{22} \geqslant 0 \quad \rho_{11} \rho_{22}-\rho_{12}^{2} \geqslant 0$

From the kinetic energy the mass matrix can be calculated. Taking the densities out of the volume integral, the following general form is obtained: 
$\mathbf{M}_{\text {porous }}=\left[\begin{array}{cc}\rho_{11} \mathbf{M} & -\rho_{a} \mathbf{M} \\ -\rho_{a} \mathbf{M} & \rho_{22} \mathbf{M}\end{array}\right]=\left[\begin{array}{cc}\rho_{1} \mathbf{M} & \mathbf{0} \\ \mathbf{0} & \rho_{2} \mathbf{M}\end{array}\right]+\rho_{a}\left[\begin{array}{cc}\mathbf{M} & -\mathbf{M} \\ -\mathbf{M} & \mathbf{M}\end{array}\right]$

If the porous matrix and the fluid have the same kind of nodal displacements, then each sub-matrix $\mathbf{M}$ is the same. It can be consistent or lumped. The first mass contribution depends on the base materials and their volume fraction, the second one on the interaction density.

\section{A porous beam and a porous displacement-based tetrahedral element}

\subsection{The porous beam element}

Beams made of a fluid-filled porous material have been modelled previously [16]. It is assumed that fluid is enclosed within the porous matrix of the beam by a thin skin. The displacements associated with the porous matrix nodes are $u_{s}, v_{s}$ and $\beta_{s}$, that is, on axis displacement, transverse displacement and transverse section rotation respectively. A linear beam with shear is assumed. For the fluid part the same displacement field is proposed, but, because of the skin, the fluid must move transversely by the same amount as the porous container. Then, $v_{f}=v_{s}$. The displacement fields are:

$$
\begin{aligned}
& u_{s}(x, y)=N_{1} u_{s}^{1}+N_{2} u_{s}^{2}-y\left(N_{1} \beta_{s}^{1}+N_{2} \beta_{s}^{2}\right) \\
& u_{f}(x, y)=N_{1} u_{f}^{1}+N_{2} u_{f}^{2}-y\left(N_{1} \beta_{f}^{1}+N_{2} \beta_{f}^{2}\right) \\
& v_{s}(x, y)=v_{f}(x, y)=N_{1} v_{s}^{1}+N_{2} v_{s}^{2}
\end{aligned}
$$

The Ns are linear Lagrangian functions of $x$. The superscript stands for the node number. The strains for the porous matrix are:

$e_{x}=e=u_{s, x}=N_{1, x} u_{s}^{1}+N_{2, x} u_{s}^{2}-y\left(N_{1, x} \beta_{s}^{1}+N_{2, x} \beta_{s}^{2}\right)$

$\gamma_{x y}=u_{s, y}+v_{s, x}=N_{1, x} v_{s}^{1}+N_{2, x} v_{s}^{2}-\left(N_{1} \beta_{s}^{1}+N_{2} \beta_{s}^{2}\right)$

and for the fluid,

$\varepsilon_{x}=\varepsilon=N_{1, x} u_{f}^{1}+N_{2, x} u_{f}^{2}-y\left(N_{1, x} \beta_{f}^{1}+N_{2, x} \beta_{f}^{2}\right)$

The beam transverse displacement does not compute volume changes in the fluid. Moreover, volume changes in the fluid and solid adopt the same functional form. A Bernoulli beam cannot be used for this model because if the solid and fluid share the same transverse displacement, they must produce the same transverse section rotation, and thus no relative fluid and solid movements can be computed under bending. The linear beam is constructed using reduced integration in the shear energy contribution [17] to avoid locking. The element stiffness matrix becomes

$$
\mathbf{K}_{\text {porous }}=\left[\begin{array}{cc}
(2 N+A) \mathbf{K}_{b}+N \mathbf{K}_{s} & Q \mathbf{K}_{b} \\
Q \mathbf{K}_{b} & R \mathbf{K}_{b}
\end{array}\right]
$$

$\mathbf{K}_{b}$ is the stiffness related to extension and bending, the one that computes unitary volume changes. $\mathbf{K}_{s}$ is the shear stiffness of the porous matrix, the one with reduced integration. The nodal displacement field is:

$\mathbf{d}^{T}=\left[\begin{array}{llllllllllll}u_{s}^{1} & v_{s}^{1} & \beta_{s}^{1} & u_{s}^{2} & v_{s}^{2} & \beta_{s}^{2} & u_{f}^{1} & v_{f}^{1} & \beta_{f}^{1} & u_{f}^{2} & v_{f}^{2} & \beta_{f}^{2}\end{array}\right]$

Because the transverse displacement of the fluid does not contribute to volume changes in the fluid, they can be eliminated, resulting in a 10 d.o.f. element. The mass matrix is composed by arranging the diagonal matrix of a linear beam as in Eq. (18).

\subsection{A four-node displacement based porous tetrahedron}

Unfortunately, a displacement-based fluid element rotation expands the null space of $\mathbf{K}_{f f}$, which the porous element will see as a kind of rigid motion. An obvious solution is to replace $\mathbf{K}_{f f}$ by a consistent acoustic fluid matrix. It must have the same nodal fluid degrees of freedom as the original matrix. The one developed in [7] is used in this paper. The displacement-based acoustic fluid proposed by Hamdi et al. [3] is also a serious candidate. In both cases the original fluid stiffness is stabilized by adding an energy related to the fluid's rotation. This energy is parameterized by the factor $\alpha$. The coefficient is not determined in Hamdi's paper. The element developed by Correa et al. [7] presents a closed form for this factor and, because it satisfies the Individual Element Test of Bergan and Hanssen [9], a different factor for each element can be used without affecting convergence. The following form for the stiffness matrix is proposed:

$\mathbf{K}_{\text {porous }}=\left[\begin{array}{cc}\mathbf{K}_{s s} & Q \mathbf{K}_{f f}^{\text {basic }} \\ Q \mathbf{K}_{f f}^{\text {basic }} & R\left(\mathbf{K}_{f f}^{\text {basic }}+\alpha \mathbf{K}_{\text {rot }}\right)\end{array}\right]$

The stiffness matrix is $\mathbf{K}_{s s}$, of a four-node linear tetrahedron [18]. The diagonal $\mathbf{K}_{f f}$ is replaced by the sum of two matrices. The matrix $\mathbf{K}_{f f}^{\text {basic }}$ is the one for computing the unit changes in the volume of the fluid and the matrix $\mathbf{K}_{\text {rot }}$ is the one in charge of computing energy under a fluid rotation. In [7] $\alpha$ is obtained using dynamic fluid behaviour considerations. No changes are needed for the out-ofdiagonal sub-matrix. They correctly compute the interaction between solid and fluid and they are not responsible for the appearance of spurious motions. A check must be made to determine whether or not the factor $\alpha$ will affect the static results in a porous material.

\section{Tetrahedral element validation}

\subsection{Minimum restrictions and consistency test}

In 1954, Biot and Willis [2] proposed two experiments to determine the coefficients proposed in their formulation. They are known as the jacketed and the unjacketed tests. In both, the specimens are subjected to a given load condition, and under this load, the porous material will undergo a constant strain state. From the strains developed in both constituents, three strains in both experiments, three equations can be constructed, yielding the coefficients $A, Q$ and $R$. Coefficient $N$ must be known in advance. These experiments are used to check the numerical consistency of our formulated element.

In the jacketed test, a sample is jacketed by a thin membrane with very low stiffness and submerged in a fluid with pressure $p$. The internal fluid is connected to the atmosphere so that no fluid stress is computed. All of the pressure acting over the sample surface is supported entirely by the solid matrix, Fig. 1a.

A computational model of this test, including the minimum degrees of freedom to simulate a constant strain state, requires careful consideration. The complete pressure load must be imposed over the six faces of a cube, affecting the solid d.o.fs with no load over the fluid ones. The minimum restrains in order to avoid rigid body motion of the solid are imposed, Fig. 2a. In this case, the corner node P will not move and the three opposite surfaces will shrink uniformly towards it. Applying the same restraints to the displacement-based fluid part will result in a singular matrix (or a badly conditioned one). It must be noted that the strain measure of the fluid is $\varepsilon=\varepsilon_{x}+\varepsilon_{y}+\varepsilon_{z}$ and then, any combination of $\varepsilon_{x}, \varepsilon_{y}$ or $\varepsilon_{z}$ can produce a result. This means that at least two values of the fluid strains must be set to zero with the appropriate constraints. In order to do so the fluid has to be encapsulated 

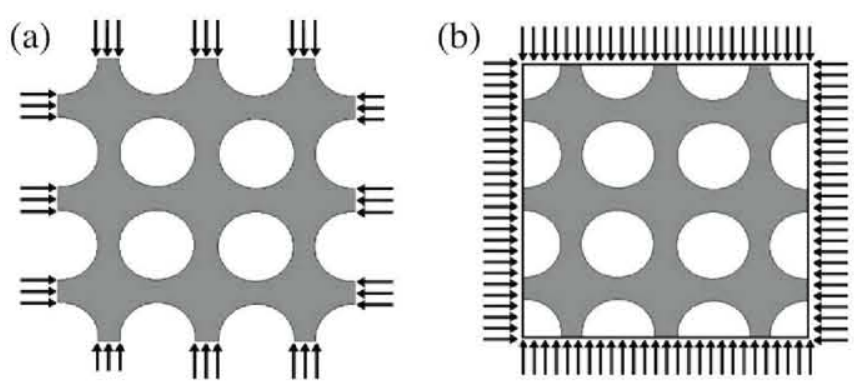

Fig. 1. Jacketed and unjacketed compressibility test. (a)

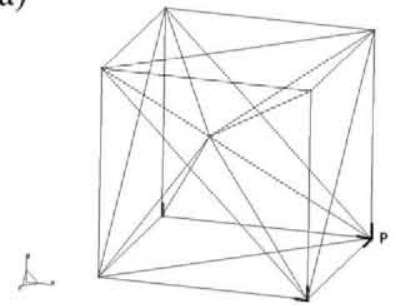

(b)

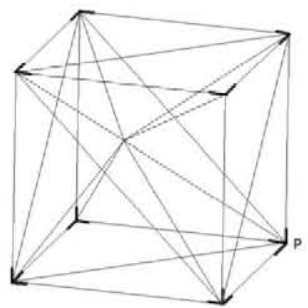

Fig. 2. Minimum cube restrictions. Solid dark line in the constrained direction. (a) Porous matrix. (b) Fluid.

in a rigid cube with a free cap. Because it is a fluid, impenetrability constraints are imposed over the side and bottom wall, Fig. 2b. A numerical model so constructed does not seem to reflect our predetermined physical idea of a jacketed test. Nevertheless, it does not violate any of the original test settings; that is, the fluid will be removed freely from the solid pore when the load is undertaken by the solid.

In the case where the elastic constants are provided, the stiffness matrix does not require explicit information about the material porosity.

From our numerical experiment, the change of volume in the solid matrix can be measured as:

$e=e_{x}+e_{y}+e_{z}$

and the so-called coefficient of jacketed compressibility can be computed

$\kappa=\frac{e}{p}$

From the constitutive equations a further useful expression is obtained:

$\frac{1}{\kappa}=\frac{2}{3} N+A-\frac{Q^{2}}{R}$

Under the continuous mechanics assumption that a positive stress will produce a positive strain, from Eq. (30) it is concluded that a valid set of elastic constants must satisfy

$\frac{2}{3} N+A \geqslant \frac{Q^{2}}{R}$

Reported experimental measurements do not check this and surprisingly draw no attention to the fact that if the elastic coefficients do not satisfy Eq. (31), the solid matrix increases its volume when loaded with an external pressure [19]. In the unjacketed test a sample is submerged in a fluid with pressure $p^{\prime}$ (see Fig. 1b). In the numerical model, with the same constraints as before, the solid matrix is loaded with a pressure $p_{s}=(1-\beta) p^{\prime}$ and the fluid with a pressure $p_{f}=\beta p^{\prime}$. It is the first time that the model is affected by the porosity. The stresses computed in the solid matrix must be the ones under a hydrostatic load:

$\sigma_{x x}=\sigma_{y y}=\sigma_{z z}=(1-\beta) p^{\prime}$

The one in the fluid is

$s=\beta p^{\prime}$

An unjacketed compressibility coefficient can be computed as:

$\delta=\frac{e}{p^{\prime}}$

Biot and Willis also defined a coefficient of fluid content for this experiment where the unit changes in the volumes of both materials are considered:

$\gamma=\frac{\beta(e-\varepsilon)}{p^{\prime}}$

Thus, three elastic coefficients can be computed from the three strains and the known value of $N$ :

$$
\begin{aligned}
& A=\frac{\frac{\gamma}{\kappa}+\beta^{2}+(1-2 \beta)\left(1-\frac{\delta}{\kappa}\right)}{\gamma+\delta-\frac{\delta^{2}}{\kappa}}-\frac{2}{3} N \\
& Q=\frac{\beta\left(1-\beta-\frac{\delta}{\kappa}\right)}{\gamma+\delta-\frac{\delta^{2}}{\kappa}} \\
& R=\frac{\beta^{2}}{\gamma+\delta-\frac{\delta^{2}}{\kappa}}
\end{aligned}
$$

The mesh in Fig. 2 for jacketed and unjacketed conditions is tested. Elastic constants are proposed for a steel matrix and a foam matrix, both with a bulk porosity of 0.95 , with air as the filling fluid (see Table 1).

The elastic properties for the foam are taken from [20]. The values for steel are obtained assuming a volume weighting of the elastic properties of a solid,

$E_{\text {porous }}=(1-\beta) E_{\text {solid }}$

Values of $R$ are approximated by the fluid bulk modulus multiplied by the porosity, and $Q$ by the bulk modulus of the fluid multiplied by the porosity squared. The Biot strains are measured in each element and averaged. From their average the elastic constants are recovered by means of Eq. (36). The computed constants match those proposed to twelve significant figures, Table 2 .

\subsection{The effect of selected porous materials and rotational stabilization on matrix conditioning}

In [7], the following closed form was obtained for the stabilization factor:

$\alpha=\frac{21}{h^{2}}+4$

where $h$, in metres, is a measure of the element size. When $h$ ranges from $1 \mathrm{~m}$ to $1 \mathrm{~cm}, \alpha$ ranges from 25 to 210,000 . The factor was obtained in order to place the spurious rotational modes at a higher

Table 1

Material properties.

\begin{tabular}{lll}
\hline Properties of porous material & Polyurethane foam and air & Steel and air \\
\hline Static Young's modulus $(\mathrm{Pa})$ & $70 \times 10^{3}$ & $1.034 \times 10^{10}$ \\
Static Poisson's ratio & 0.38 & 0.3 \\
Porous material density $\left(\mathrm{kg} / \mathrm{m}^{3}\right)$ & 22.1 & 7850 \\
Porosity & 0.95 & 0.95 \\
Air density $\left(\mathrm{kg} / \mathrm{m}^{3}\right)$ & 1.20 & 1.20 \\
Air compressibility $(\mathrm{Pa})$ & $1.42 \times 10^{5}$ & $1.42 \times 10^{5}$ \\
\hline
\end{tabular}


Table 2

Proposed and computed coefficients.

\begin{tabular}{lll}
\hline Coefficients & Proposed $(\mathrm{Pa})$ & Obtained $(\mathrm{Pa})$ \\
\hline Foam air porous & material & \\
$N$ & 25362.31884057972 & Not computed \\
$A$ & $80314.0096618 \underline{3575}$ & $80314.0096618 \underline{5253}$ \\
$Q$ & $64077.5000000 \underline{0000}$ & $64077.500000 \underline{\underline{4686}}$ \\
$R$ & $134900.000000 \underline{0000}$ & $134900.000000 \underline{1225}$ \\
Steel and air porous & material & \\
$N$ & 3976923076.923077 & Not computed \\
$A$ & $5965384615.3846 \underline{15}$ & $5965384615.3846 \underline{21}$ \\
$Q$ & $64077.5000000 \underline{0000}$ & $64077.5000000 \underline{6797}$ \\
$R$ & $134900.000000 \underline{0000}$ & $134899.999999 \underline{8490}$ \\
\hline
\end{tabular}
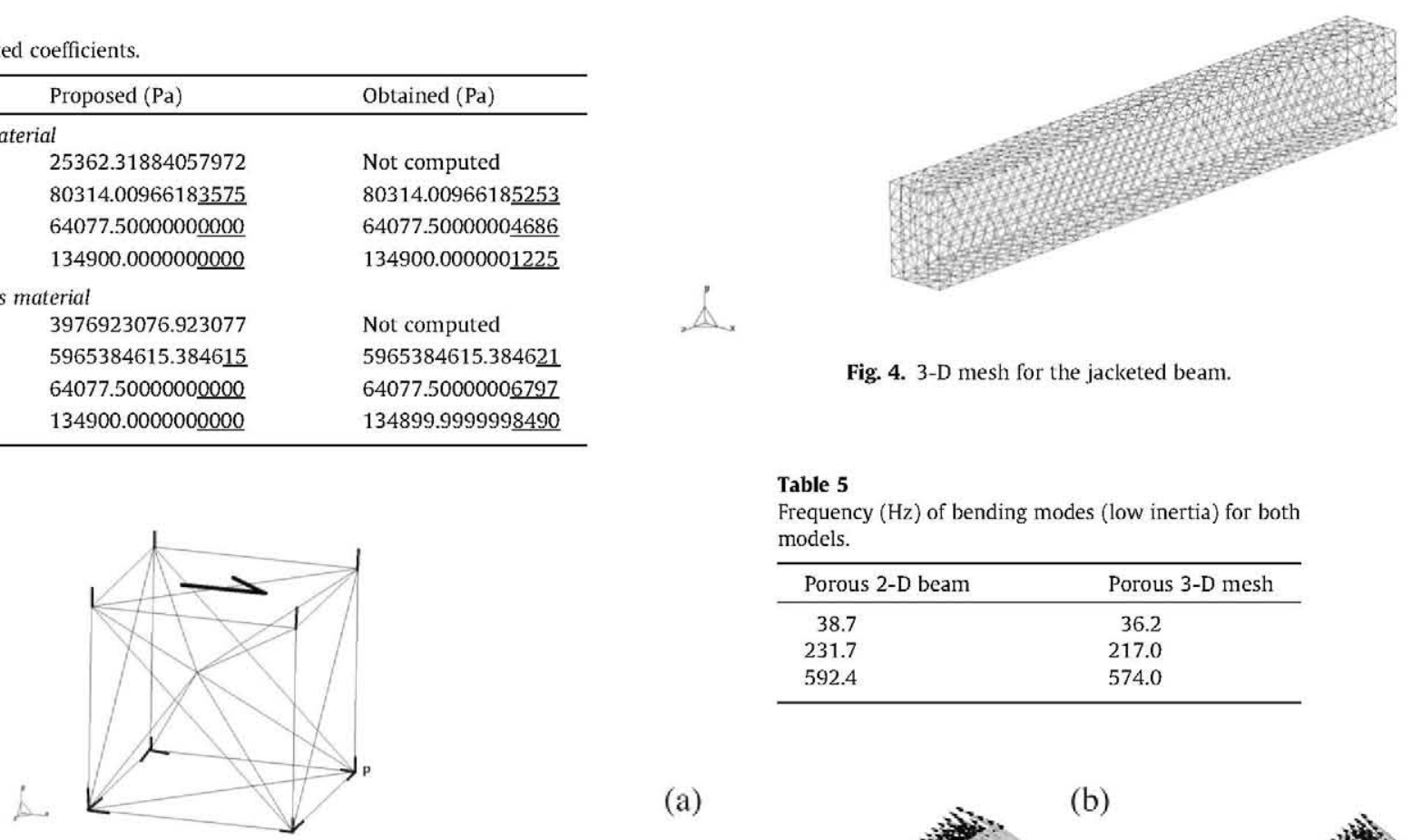

Fig. 4. 3-D mesh for the jacketed beam.

Table 5

Frequency $(\mathrm{Hz})$ of bending modes (low inertia) for both models.

\begin{tabular}{cc}
\hline Porous 2-D beam & Porous 3-D mesh \\
\hline 38.7 & 36.2 \\
231.7 & 217.0 \\
592.4 & 574.0 \\
\hline
\end{tabular}

(a)

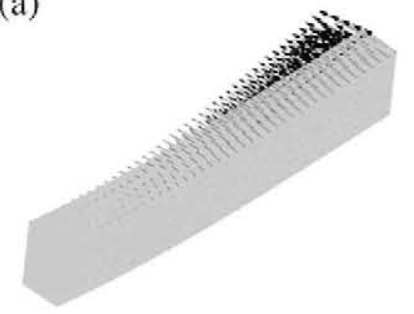

(b)

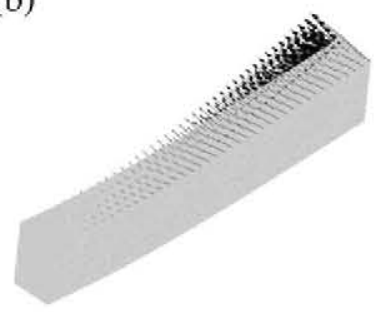

Table 3

Stiffness matrix condition and stabilization factor for two porous materials as a function of mesh size.

\begin{tabular}{llll}
\hline Mesh size $(\mathrm{m})$ & $d=1$ & $d=0.01$ & $d=0.001$ \\
\hline Foam-air & & & \\
Matrix condition & $3.3249 \mathrm{e}+05$ & $5.6687 \mathrm{e}+05$ & $5.4981 \mathrm{e}+06$ \\
Penalty factor $\alpha$ & 25.000 & $2.1000 \mathrm{e}+05$ & $2.1000 \mathrm{e}+07$ \\
Steel-air & & & \\
Matrix condition & $2.6996 \mathrm{e}+05$ & $2.9397 \mathrm{e}+05$ & $3.3346 \mathrm{e}+05$ \\
Penalty factor $\alpha$ & 25.000 & $2.1000 \mathrm{e}+05$ & $2.1000 \mathrm{e}+07$ \\
\hline
\end{tabular}

frequency than the higher longitudinal mode the mesh was able to capture adequately. How will this affect matrix conditioning in the solution of static porous problems? How will this factor affect a numerical model that may already be ill conditioned by the material properties? A low value of $\alpha$ will produce a quasi-singular stiffness matrix if the shear deformation of the fluid is not restrained. A high value of $\alpha$ can produce a fluid shear constraint that obscures the normal compressive fluid behaviour.

Because the coefficient computed in Eq. (38) is designed to penalize rotational fluid behaviour, a simple shear test is proposed, Fig. 3. It is a cube of porous material with a filling fluid. A shearing force is applied over the upper surface of the porous matrix. The fluid and solid are completely restrained to move at the cube base.

Fig. 5. 3-D model flexural mode at $36.2 \mathrm{~Hz}$. (a) Porous matrix displacement vector. (b) Fluid displacement vector. (a)

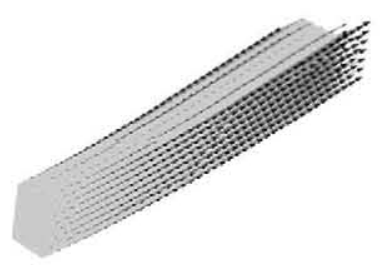

(b)

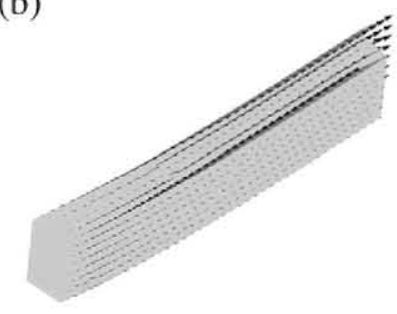

Fig. 6. 3-D model flexural mode at $36.2 \mathrm{~Hz}$. (a) Porous matrix on-axis displacement vector component. (b) Fluid on-axis displacement vector component.

Displacements of both materials in the $y$ direction are constrained at the top and the node inside is left to move freely for both materials. The idea behind the test is that if the element formulation is

Table 4

Stresses and strains for the shear test.

\begin{tabular}{llll}
\hline Mesh size & $d=1$ & $d=0.01$ & $d=0.001$ \\
\hline Foam-air & & & \\
Stress solid $\tau_{x y}$ & 0.006000000000000 & 60.000000000000036 & 600.0000000000001 \\
Stress fluid $s$ & 0 & 0 & 0 \\
Strain fluid $\varepsilon_{f}$ & 0 & 0 & 0 \\
Steel-air & & & \\
Stress solid $\tau_{x y}$ & 6000.000000000004 & 60000000.00000007 & 6000000000.000016 \\
Stress fluid $s$ & 0 & 0 & 0 \\
Strain fluid $\varepsilon_{f}$ & 0 & 0 & 0 \\
\hline
\end{tabular}




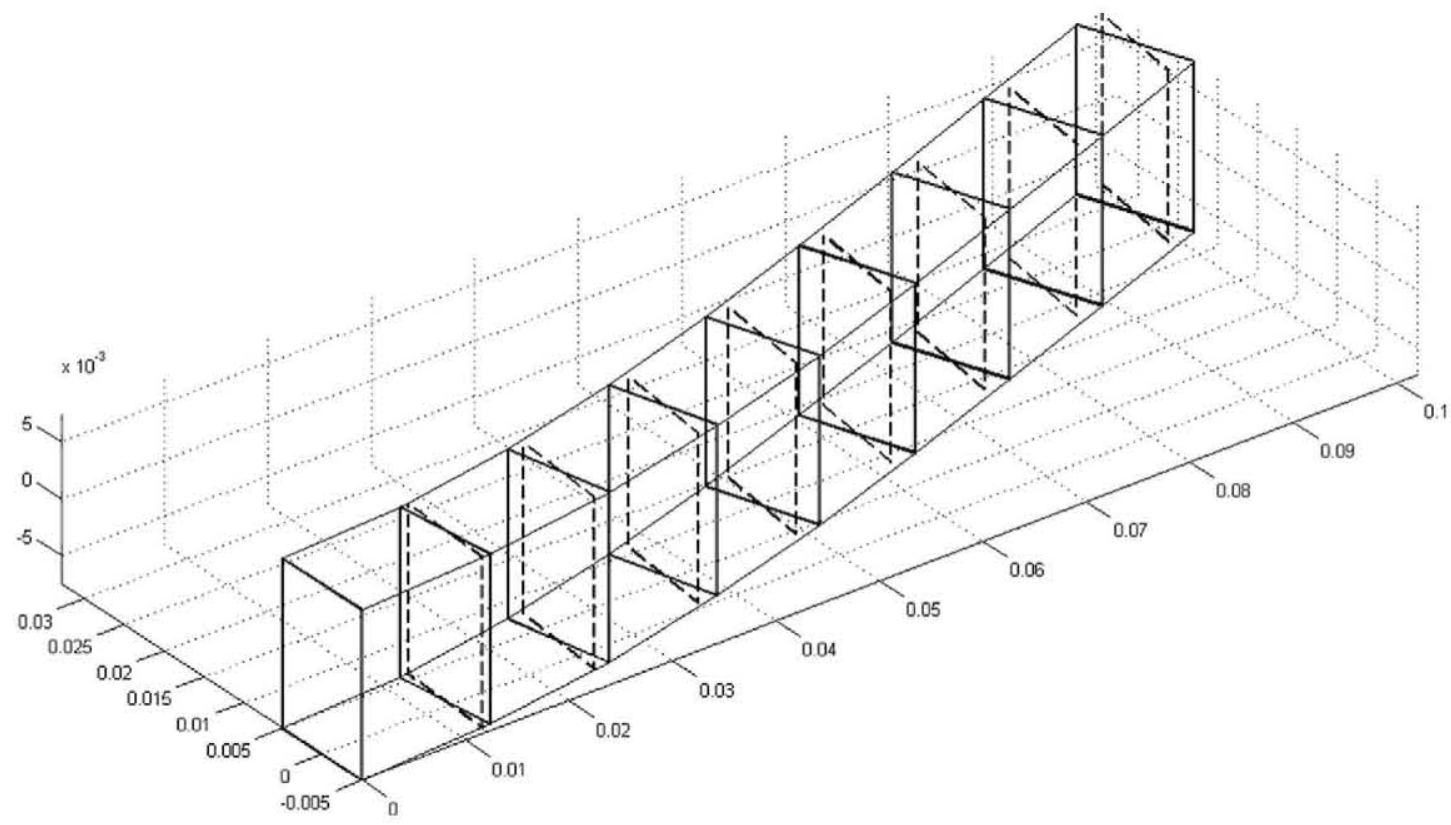

Fig. 7. Beam flexural mode, $38.7 \mathrm{~Hz}$.

not adequate, the shear in the solid will transmit volumetric strains to the fluid. Moreover, the high value of $\alpha$ coefficient can also degenerate the nature of the solution. Three meshes are run with cube sides $d$ of $1,0.01$ and $0.001 \mathrm{~m}$.

A shear stress of $0.006 / d^{2} \mathrm{~Pa}$ is applied for the foam-air pair and a value of $6000 / d^{2} \mathrm{~Pa}$ for the steel-air. Under this load, the solid should undergo a pure shear deformation and the fluid should remain unstressed. For the steel-air pair, Table 3, the factor $\alpha$ increases by six orders of magnitude but the condition number remains the same order of magnitude for the three mesh sizes. The effect of the penalty factor on the matrix condition cannot be traced. Shear stresses are recovered up to twelve significant figures, Table 4 . The software is run under the MATLAB environment in double precision ( 64 bits). The values for the fluid strain and stress are zero within machine precision.

\section{Dynamic behaviour}

The dynamic behaviour of the model is checked by simulating a rectangular beam made of porous material. Its elastic properties are shown in Table 1, column 1. The beam is assumed jacketed so that the beam hypotheses used in S3.1 are not violated. The jacketed condition is that the fluid over the boundaries must move the same amount as the porous material does in the direction normal to the beam lateral surfaces. The same beam of dimensions $0.01 \times 0.015 \times 0.10 \mathrm{~m}$ is modelled with the porous tetrahedron and with the plane linear beam.

The 3-D model needs at least four elements on the shortest side to converge to the lowest bending frequencies, Fig. 4. Only the first three bending modes associated with the lower inertia are compared. For these modes it can be considered that the section of the beam remains rectangular. Longitudinal frequencies are not compared because the 3-D model exhibits a pronounced stretching of the transverse area. The plane beam model was run with the small inertia value.

The frequencies match to within $6 \%$, Table 5 . The 2 -D beam model is stiffer. In Figs. 5 and 6 the 1st flexural mode is depicted. In the 3-D model, the transverse displacement vectors and their on-axis component are shown for the porous material and the fluid. From the on axis displacements it can be concluded that the hypothesis of a neutral bending axis is adequate. For the beam element, Fig. 7, the transversal section of the fluid (dashed lines) and the porous matrix (solid lines) are shown together. As in the 3-D case, it can be seen that when the porous material is compressed, the fluid is expelled from the pores.

\section{Conclusions}

In this work, starting from Biot's elastic energy formulation, and assuming that a suitable acoustic fluid behaviour can be formulated with the same polynomial expansion and degrees of freedom as a solid elastic element, a simple stiffness matrix for a displacement-based porous material is constructed. As a matter of fact, only two different basic matrices need to be constructed - one for the elastic behaviour of the porous material and another that computes the stiffness associated with the acoustic fluid's changes in volume. For cases of a porous bar or a porous plane beam where no spurious rotational modes can arise, the formulation is effective. In the case where a planar or a 3-D element is going to be constructed, it is necessary to replace the fluid diagonal sub-matrix by one which is free of spurious rotational modes. Candidates are the elements developed by Hamdi et al. [3] and Bathe et al. $[4,21]$. Raviart-Thomas elements are disregarded only because of their awkward node location, but they fit if an elastic solid can be developed with the same degrees of freedom and polynomial order of the fluid one.

In order to honour Biot's experimental proposal to compute the elastic constants of a porous material, a cube with a free node inside, as well as minimal restrictions, are used to check the consistency of the formulation. This is simply Bruce Iron's Patch Test [22] and because the element is formulated so as to pass the IET of Bergan and Hanssen [9], forms A, B and C proposed by Taylor et al. [23] are also satisfied as demonstrated in [24]. Basically three constant strain measures are combined to obtain three elastic coefficients, in keeping with Biot's formulation. If the element fails to satisfy Biot's formulation, then the elastic coefficients used in the model are assumed to be unrecoverable. The element has also shown to be consistent for very extreme porous material properties.

Because the stabilization factor, originally developed under dynamic considerations, changes many orders of magnitude 
depending on element size, a shear test was proposed to ascertain the extent to which the numerical condition deteriorates, or even worse, obscures the ability of the element to compute fluid volume changes. The test showed that the condition number does not change with mesh size and that a porous solid matrix under shear will not produce fluid volume changes for any of the cases tested.

Finally, a simple dynamic problem was run to mutually check the 3-D and the beam formulations. A comparison was made only for the modes where the jacketed beam approximation was reasonable. In those cases, the frequencies and modal shapes matched. The beam model seems adequate to model narrow tubes filled with porous materials.

\section{References}

[1] Biot MA. General theory of three dimensional consolidation. J Appl Phys $1941 ; 12: 155-64$

[2] Biot MA, Willis DG. The elastic coefficients of the theory of consolidation. J Appl Mech 1954;24:594-601.

[3] Hamdi MA et al. A displacement method for the analysis of vibrations of coupled fluid-structure systems. Int J Numer Methods Eng 1978;13:139-50.

[4] Bathe KJ, Nitikitpaiboon C, Wang X. A mixed displacement-based finite element formulation for acoustic fluid-structure interaction. Comput Struct 1995;56:225-37.

[5] Bermúdez A, Hervella-Nieto L, Rodríguez R. Finite element computation of threedimensional elastoacoustic vibrations. J Sound Vib 1999;219(2):279-306.

[6] Aneiros Andrés Prieto. Some contributions in time-harmonic dissipative acoustic problems harmonic. $\mathrm{PhD}$ thesis, Universidad de Santiago de Compostela; 2005. p. 44-57.

[7] Correa S, Militello C, Recuero M. Acoustic displacement tetrahedral developed using the IET rules. Comput Struct 2010;88:985-90.

[8] Felippa CA, Militello C. Variational formulation of high performance finite elements: parametrized variational principles. Comput Struct 1990;36:1-11.
[9] Bergan PG, Hanssen L. A new approach for deriving "Good" finite elements. In: Whiteman JR, editor. The mathematics of the finite element, vol. II. London: Academic Press; 1975.

[10] Ghaboussi J, Wilson EL. Variational formulation of dynamics of fluid saturated porous elastic solids. Proc ASCE 1972;98(EM4):947-63.

[11] Panneton R, Atalla N. An efficient finite element scheme for solving the threedimensional poroelasticity problem in acoustics. J Acoust Soc Am 1997;101(6):3287-98.

[12] Morand $H$, Ohayon R. Fluid-structure interaction. Applied numerical methods. Wiley; 1995

[13] Göransson P. Acoustic finite element formulation of a flexible porous material. A correction for inertial effects. J Sound Vib 1995;185(4):559-80.

[14] Göransson P. A weighted residual formulation of the acoustic wave propagation through a flexible porous material and a comparison to a limp material model. J Sound Vib 1995;182(3):479-94.

[15] Chen HC, Taylor RL. Vibration analysis of fluid-solid system using a finite element displacement formulation. Int J Numer Methods Eng 1990;29: 683-98.

[16] Cederbaum G, Li L, Schulgasser K. Poroelastic structures. Oxford: Elsevier Science; 2000.

[17] Hughes TJR. The finite element method linear static and dynamic finite. Englewood Cliffs, NJ: Prentice-Hall; 1987.

[18] Zienkiewicz OC, Taylor RL. The finite element method Vol I: the basis. Oxford: Butterworth-Heinemann; 2000.

[19] Kubik J, Kachmaryk M, Chaplya E. Methods for the determination of the characteristics of porous saturated media. Mater Sci 2001;37:92-102.

[20] Hörlin Nils-Erik. 3D hierarchical hp-FEM applied to elasto-acoustic modeling of layered porous media. J Sound Vib 2005;285:341-63.

[21] Wang X, Bathe KJ. Displacement/pressure based finite element formulations for acoustic fluid-structure interactions. Int J Numer Methods Eng 1997;40:2011-7.

[22] Irons B, Ahmad S. Techniques of finite elements. West Essex, England: Ellis Horwood Limited Publishers; 1979.

[23] Taylor RL, Simo JC, Zienkiewicz OC, Chan ACH. The patch test - a condition for assessing FEM convergence. Int J Numer Methods Eng 1986;22:39-62.

[24] Militello C, Felippa CA. The individual element test revisited. In: Oñate E. et al. editors. The finite element in the 1990's. Springer-Verlag; 1991. 